\title{
Os ramos colaterais da aorta abdominal em jaguatirica (Leopardus pardalis) ${ }^{1}$
}

\author{
Luane L. Pinheiro ${ }^{2 *}$, Elenara B. Araújo², Ana Rita de Lima², Danielli M. Martins², \\ Raysa Melul'², Ana Carla B. Souza ${ }^{3}$, Luiza C. Pereira ${ }^{3}$ e Érika Branco ${ }^{2}$
}

\begin{abstract}
Pinheiro L.L., Araújo E.B., Lima A.R., Martins D.M., Melul R., Souza A.C.B., Pereira L.C. \& Branco E. 2014. [The collateral branches from abdominal aorta in ocelot (Leopardus pardalis).] Os ramos colaterais da aorta abdominal em jaguatirica (Leopardus pardalis). Pesquisa Veterinária Brasileira 34(5):491-495. Laboratório de Pesquisa Morfológica Animal, Universidade Federal Rural da Amazônia, Av. Presidente Tancredo Neves 2501, Belém, PA 66077-530, Brazil. E-mail: luanelopespinheiro@gmail.com

The ocelot (Leopardus pardalis) is a species of wild cat that little has been investigated by their morphology. Thus, the study aimed to detail the origin and distribution of collateral branches from abdominal aorta this animal. We evaluated two exemplary, one male and one female, young, from Paragominas-PA, donated to Animal Morphology Research Laboratory (LaPMA) Federal Rural University of Amazonia (UFRA). The arterial system was filled with red latex and the bodies were preserved with buffered formaldehyde solution 10\%. The abdominal aorta of L. pardalis originated between T12 and L1, and the celiac artery was first visceral branch in the craniocaudal direction, resulting in the hepatic a., gastric left a. and splenic a.. The caudal pancreaticoduodenal a., ileal aa., ileocolic aa., right and left renal aa., right and left adrenal aa., and. right and left ovarian aa. or testicular aa. were founded after. Parietally the abdominal aorta yielded an average of six lumbar branches, as well as frenicoabdominal aa., deep circumflex iliac aa. and external and internal iliac aa.. The abdominal aorta still originated the caudal mesenteric a., which is divided into the left colic a. and the cranial rectal a.. The left colic artery followed cranially parallel to the descending colon irrigating it, originating an average of 18 branches, and anastomosing with middle colic a.. The cranial rectal artery followed caudally emerging into eight branches uniting with the final portion of the descending colon and the rectum, together with the rectal middle a.. Finally, the abdominal aorta emerged as the terminal branch, the median sacral. The abdominal arterial vascularization of the ocelot is quite similar to that described in domestic cats, with differences in the number of jejunal arteries and origin of the renal arteries. The abdominal arterial vasculature of this species is quite similar to that described in domestic cats and other mammals, differing in number of the jejunal arteries and origin of the renal arteries.
\end{abstract}

INDEX TERMS: Leopardus pardalis, anatomy, aorta, artery, wild cat.

\footnotetext{
${ }^{1}$ Recebido em 23 de janeiro de 2014.

Aceito para publicação em 27 de março de 2014.

${ }^{2}$ Laboratório de Pesquisa Morfológica Animal (LaPMA), Universidade Federal Rural da Amazônia (UFRA), Av. Presidente Tancredo Neves 2501, Belém, PA 66077-530, Brasil. *Autor para correspondência: luanelopespinheiro@gmail.com

${ }^{3}$ Mina de Bauxita Paragominas, Empresa Terra Ltda, Estrada da Mineração s/n, Rodovia BR-010 Km 30, Paragomina, PA 68625-970, Brasil. E-mails: elenarabotelho@gmail.com, vetlima@uol.com.br, daniellimartinelli@gmail.com, raysamelul@gmail.com, luiza.lpcv@hotmail.com, ebranco.ufra@gmail.com
}

RESUMO.- A jaguatirica (Leopardus pardalis) é uma das espécies de felino silvestre que pouco foi investigada quanto a sua morfologia. Assim, o estudo objetivou detalhar a origem e distribuição dos ramos colaterais da aorta abdominal deste animal. Avaliou-se dois exemplares, sendo um macho e uma fêmea, jovens, provenientes de Paragominas-PA, doados ao Laboratório de Pesquisa Morfológica Animal (LaPMA) da Universidade Federal Rural da Amazônia (UFRA). 0 sistema arterial foi preenchido com látex pigmentado de vermelho e os cadáveres foram preservados 
com solução de formaldeído tamponado a $10 \%$. A aorta abdominal do L. pardalis teve origem entre T12 e L1, sendo a artéria celíaca o primeiro ramo visceral no sentido crânio-caudal, resultando nas artérias hepática, gástrica esquerda e esplênica. A artéria mesentérica cranial surgiu como segundo ramo da aorta abdominal, originando as artérias jejunais. Na sequência localizamos artéria pancreáticoduodenal caudal, artérias ileais, artérias ileocólicas, artérias renais direita e esquerda, artérias adrenais direita e esquerda e artérias ováricas ou testiculares direita e esquerda. Parietalmente, a aorta abdominal originou em média seis ramos lombares, bem como a artéria frenicoabdominal, as artérias circunflexas ilíacas profundas e artérias ilíacas externa e interna. A aorta abdominal gerou ainda a artéria mesentérica caudal, a qual dividiu-se em artérias cólica esquerda e retal cranial. A artéria cólica esquerda seguiu cranialmente paralela ao cólon descendente irrigando-o, originando em média 18 ramos, e anastomosando-se com a artéria cólica média. A artéria retal cranial seguiu em direção caudal distribuindo oito ramos à porção final do cólon descendente e ao reto, e uniu-se com a artéria retal média. Por fim, a aorta abdominal emitiu como ramo terminal a artéria sacral mediana. A vascularização arterial abdominal desta espécie é bastante semelhante ao descrito em felinos domésticos e demais mamíferos, com diferenças quanto ao número de artérias jejunais e origem das artérias renais.

TERMOS DE INDEXAÇÃO: Leopardus pardalis, anatomia, aorta, artéria, gato selvagem.

\section{INTRODUÇÃo}

A Jaguatirica (Leopardus pardalis Linnaeus, 1758) é o terceiro maior felino das Américas, considerado de médio porte, com peso variando entre 11 e $16 \mathrm{~kg}$ (Murray \& Gardner 1997). No Brasil pode ser vista em ecossistemas variados, de florestas úmidas à caatinga, ocorrendo em todos os estados com exceção provável do Rio Grande do Sul (Oliveira \& Cassaro 1999).

São animais solitários, estritamente carnívoros, possuem hábitos diurnos e noturnos, sendo mais ativos durante a noite (Emmons \& Feer 1990). Possui pelo denso e curto de cor amarelada, com pintas escuras em todo o corpo, exceto na região ventral, que é esbranquiçada (Oliveira \& Cassaro 1999). A jaguatirica, assim como outras seis espécies de felinos brasileiros, está incluída na lista de espécies da fauna brasileira ameaçadas de extinção (IUCN 2013).

A aorta abdominal, é parte da aorta descendente que penetra na cavidade abdominal após atravessar o hiato aórtico do diafragma (Ghoshal 1986). Seus ramos colaterais estão distribuídos pela parede e conteúdo da cavidade abdominal, medula espinhal e outros se estendem para dentro da pelve e escroto (König \& Liebich 2011).

Diante da escassa literatura sobre os aspectos morfológicos de Leopardus pardalis e em que pese sua importância como integrante de ecossistemas brasileiros, que permanece sem um padrão anatômico conhecido, o presente estudo objetivou investigar e descrever os ramos colaterais da aorta abdominal nesta espécie.

\section{MATERIAL E MÉTODOS}

Foram estudados dois exemplares de jaguatirica (Leopardus pardalis), sendo um macho e uma fêmea, ambos jovens, que foram a óbito por atropelamento, provenientes de Paragominas-PA, Brasil/Empresa Terra LTDA, sob autorização SEMA-PA no-485/2009 e 522/2009 e doados ao Laboratório de Pesquisa Morfológica Animal (LaPMA) da Universidade Federal Rural da Amazônia (UFRA).

Os animais foram criopreservados e posteriormente descongelados em água corrente por um período de duas horas. Inicialmente realizou-se a canulação da artéria carótida comum esquerda, perfundindo-se látex Neoprene 650, corado com pigmento vermelho $\left(\mathrm{Xadrez}^{\circledR}\right.$ ) em todo sistema circulatório arterial dos animais. Imediatamente prosseguiu-se com a fixação dos cadáveres utilizando solução aquosa de formaldeído tamponado a $10 \%$ por meio de infusão intramuscular, subcutânea e intracavitária. Posteriormente, os animais foram submersos na mesma solução fixadora, onde permaneceram por sete dias.

Com o uso de materiais básicos de dissecação, realizou-se incisão na cavidade abdominal de cada animal, permitindo acesso a aorta abdominal e expondo-se a origem de todos os seus ramos.

Toda nomenclatura adotada foi baseada na Nomenclatura Atômica Veterinária (International Committee on Veterinary Gross Anatomical Nomenclature 2012).

\section{RESULTADOS}

A aorta abdominal de Leopardus pardalis apresentou origem entre T12 e T13 no macho e entre T13 e L1 na fêmea, com peso aproximado de $15 \mathrm{~kg}$ em ambos os animais, emergindo na cavidade abdominal após atravessar o hiato aórtico entre os pilares do diafragma, e emitindo ventral e sequencialmente, a artéria celíaca e a artéria mesentérica cranial. A artéria celíaca foi o primeiro ramo visceral, no sentido crânio-caudal, curta e de grosso calibre, e apresentou três ramos com mesma origem: a artéria hepática, a artéria gástrica esquerda e a artéria esplênica (Fig.1).

A artéria mesentérica cranial, apresentou-se como sendo o vaso de maior calibre (Fig.1), surgiu caudal à artéria celíaca e seguiu em direção ao mesentério originando as artérias ileocólicas, artéria pancreaticoduodenal caudal, que se dividiu em dois ramos (direito e esquerdo), artérias jejunais, que totalizaram em 12 ramos em ambos os animais, e artérias ileais, que confluíam em direção ao íleo e ao ceco (Fig.2). Na fêmea observou-se que a primeira artéria jejunal se originou juntamente com a artéria pancreaticoduodenal caudal.

Caudalmente à origem da artéria mesentérica cranial, a aorta abdominal emitiu as artérias adrenais, uma direita e outra esquerda (Fig.1). Em seguida, surgiram às artérias renais direita e esquerda, ambas calibrosas, sendo que a direita dispôs-se ligeiramente mais cranial que a esquerda no macho, estando em conformidade com a posição mais cranial do rim direito, enquanto que na fêmea a artéria renal esquerda projetou-se mais cranialmente que a direita. Ambas emitiram pequenos ramos colaterais em direção a gordura perineal (Fig.1 e 2).

As artérias testiculares e ováricas de L. pardalis, tanto direita quanto esquerda, apresentaram-se longas e delgadas, e surgiram ventrolateralmente da abdominal com certo distanciamento entre si, sendo a esquerda mais cranial 


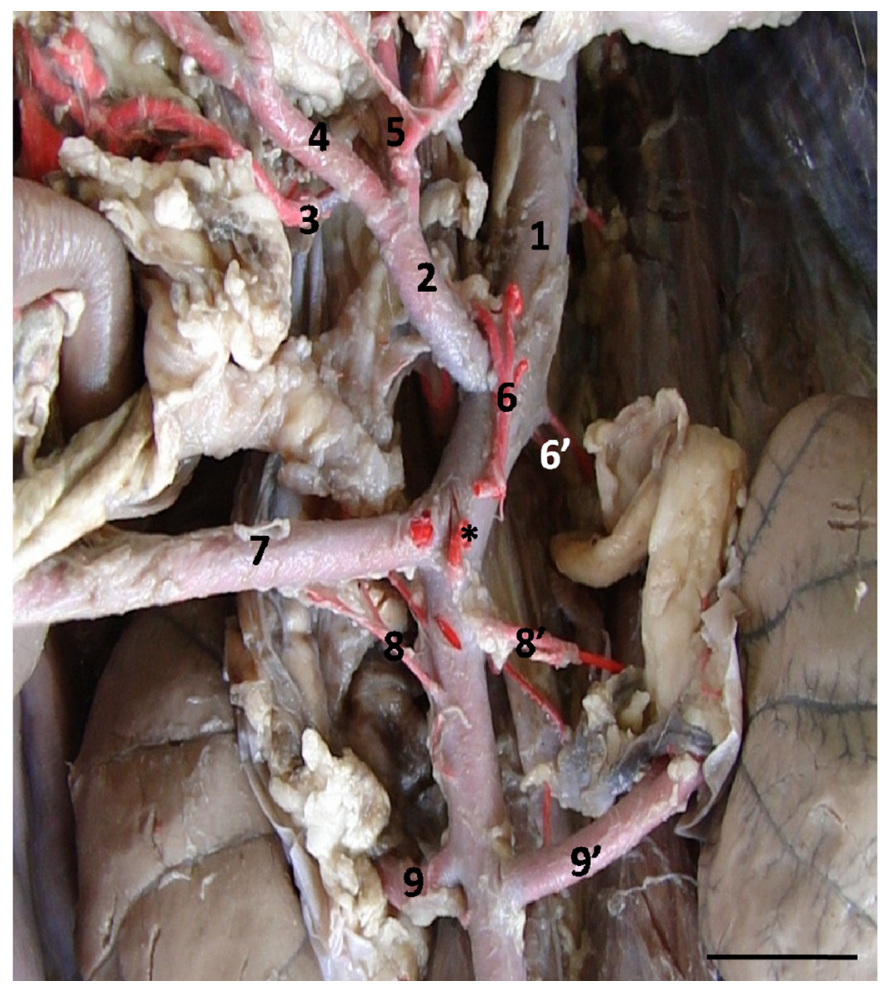

Fig.1. Vista ventral da porção cranial da aorta abdominal mostrando os ramos colaterais do exemplar macho de jaguatirica (Leopardus pardalis). Podem ser observadas: aorta abdominal (1), a. celíaca (2), a. gástrica esquerda (3), a. esplenica (4), a. hepática (5), a. frenicoabdominal direita (6) e sua origem $\left({ }^{*}\right)$, a. frenicoabdominal esqueda (6'), a. mesentérica cranial (7), aa. adrenais direita e esquerda (8 e 8') e aa. renais direita e esquerda (9 e 9'). Barra de escala: $1 \mathrm{~cm}$.

em relação à direita no macho, ocorrendo o inverso na fêmea (Fig.2 e 3).

Em seguida destacou-se a artéria mesentérica caudal, a qual teve origem na face ventral, próximo a porção terminal da aorta abdominal, percorrendo trajeto crânio-caudal, dando origem à artéria cólica esquerda e à artéria retal cranial (Fig.2 e 3).

Constatou-se que a artéria cólica esquerda dirigiu-se cranialmente arqueando-se paralelamente ao cólon descendente, dividindo-se em 18 ramos. Estes, por sua vez, originaram novos sub-ramos, que penetraram na superfície do cólon descendente, irrigando-o. Posteriormente, a artéria cólica esquerda anastomosou-se com a artéria cólica média, um ramo da artéria mesentérica cranial (Fig.2). Já a artéria retal cranial percorreu em direção caudal distribuindo em média oito ramos à porção final do cólon descendente e ao reto, até anastomosar-se com a artéria retal média, proveniente da artéria pudenda interna.

A aorta abdominal emitiu alguns ramos parietais em ambos os animais, sendo inicialmente registrada a artéria frênicoabdominal, que é par e remeteu-se entre as artérias mesentérica cranial e renais. A artéria frênicoabdominal esquerda partiu lateralmente, e a direita ventralmente da aorta abdominal, em ambos os exemplares (Fig.1).

Em média, seis ramos lombares surgiram da superfície dorsal da aorta abdominal. Lateralmente, e em par, tam-

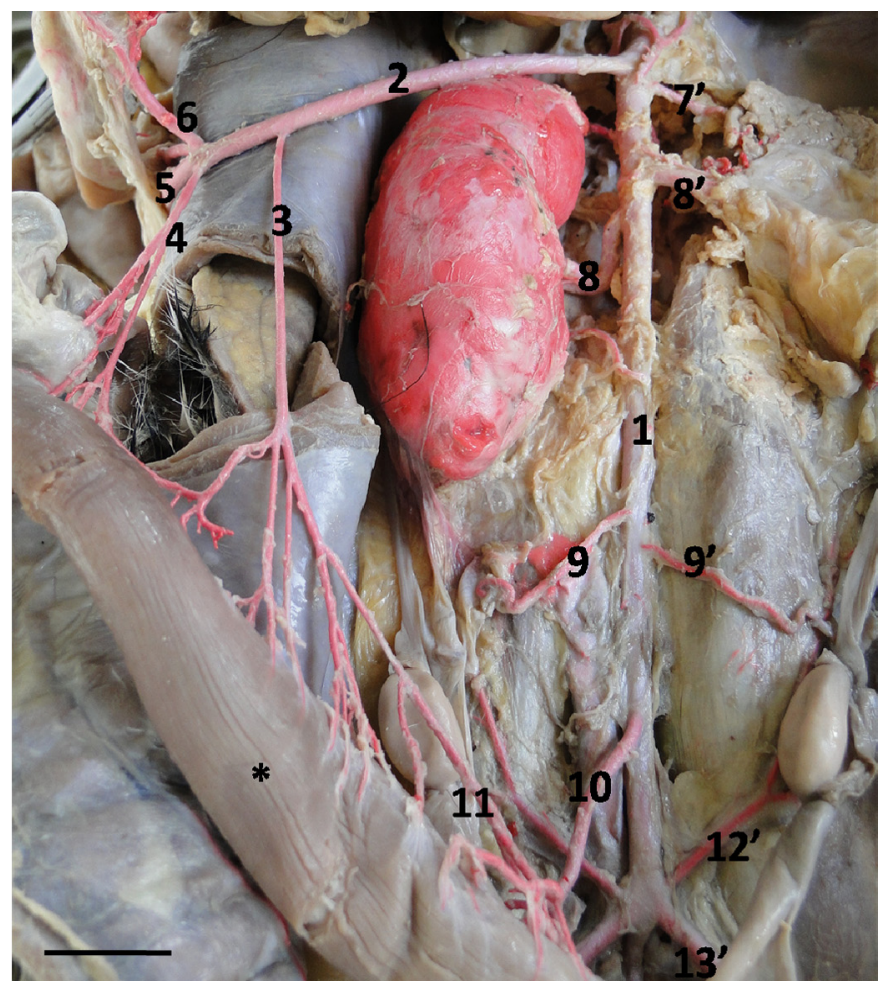

Fig.2. Vista ventral da aorta abdominal do exemplar fêmea de jaguatirica (Leopardus pardalis). Podem ser observadas: aorta abdominal (1), mesentérica cranial (2), a. cólica média (3), a. ileocólica (4), a. jejunal (5), a. pancreáticoduodenal (6), a. frênicoabdominal esquerda ( 7 '), aa. renais direita e esquerda (8 e 8'), aa. ovarianas direita e esquerda (9 e 9'), a. mesentérica caudal (10), a. cólica esquerda (11), a. circunflexa liíaca profunda esquerda (12'), a. ilíaca externa esquerda (13'), a. ilíaca interna esquerda (15') e colón descendente $\left(^{*}\right)$. Barra de escala: $1 \mathrm{~cm}$.

bém originou-se da aorta abdominal a artéria circunflexa ilíaca profunda. e em seguida, esta emitiu ainda as artérias ilíacas externas e internas, sendo estes os ramos terminais, juntamente com a artéria sacral mediana (Fig.2 e 3).

A artéria ilíaca externa surgiu como ramo na face lateral da aorta abdominal e cranial à origem da artéria ilíaca interna, que por sua vez, originou-se da bifurcação da aorta que seguiu como artéria sacral mediana (Fig.3).

\section{DISCUSSÃO}

A aorta abdominal de Leopardus pardalis apresentou origem, de forma geral, entre T12 e L1, emergindo na cavidade abdominal após atravessar o hiato aórtico entre os pilares do diafragma, e emitindo em sequência, a artéria celíaca e a artéria mesentérica cranial, ventralmente. Essas artérias originam-se independentemente uma da outra em gatos, suínos, coelhos e cães, mas por tronco comum em ovinos (Ghoshal 1986, König \& Leibich 2004). Porém, há registros no cão, de que pode haver a formação de um tronco celíaco-mesentérico, de forma que ambas as artérias (celíaca e mesentérica cranial) teriam uma única origem (Schmidt et al., 2007), o que também é descrito em felinos domésticos (Magno et al., 2009), fato não registrado em L. pardalis.

A artéria celíaca da jaguatirica é o primeiro ramo vis- 


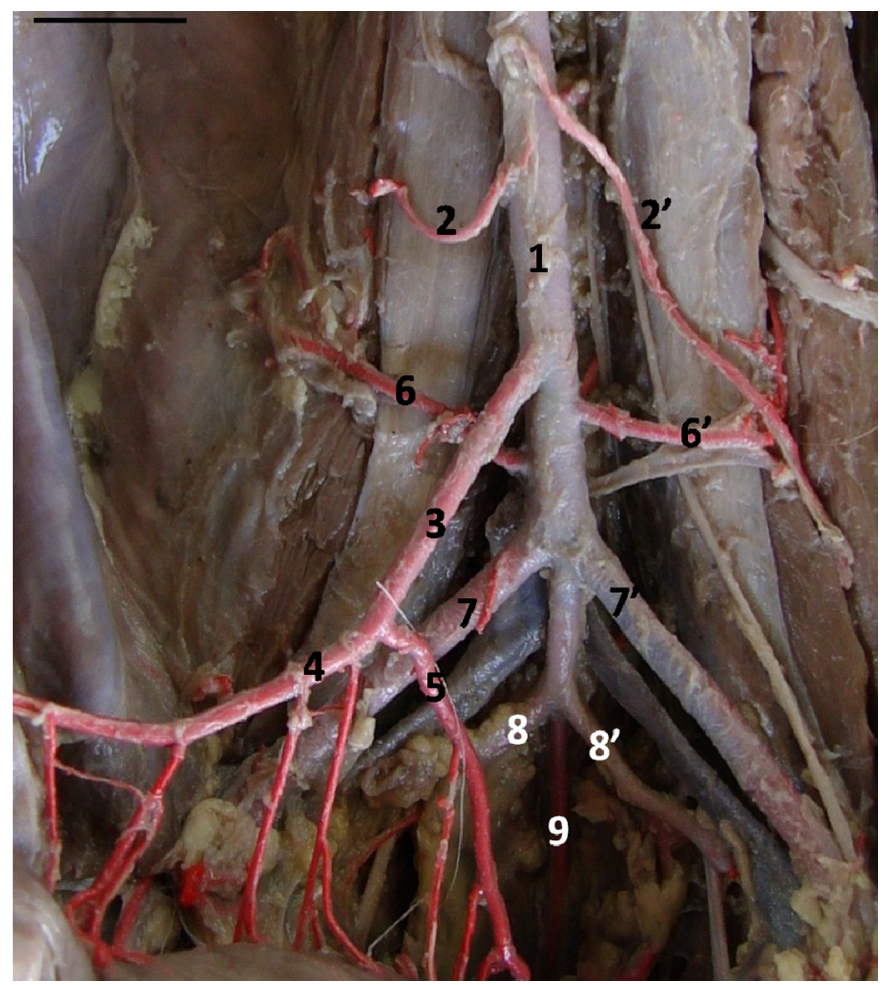

Fig.3. Vista ventral da porção caudal da aorta abdominal do exemplar macho de jaguatirica (Leopardus pardalis), mostrando os ramos colaterais e terminais. Podem ser observadas: aorta abdominal (1), aa. testiculares direita e esquerda (2 e 2'), a. mesentérica caudal (3), a. cólica esquerda (4), a. cranial retal (5), aa. circunflexa ilíaca profunda direita e esquerda (6 e 6'), aa. ilíacas externas direita e esquerda (7 e 7'), aa. ilíacas internas direita e esquerda (8 e 8') e a. sacral mediana (9). Barra de escala: $1 \mathrm{~cm}$.

ceral, no sentido crânio-caudal. É curta, de grosso calibre e apresenta três ramos com mesma origem: a artéria hepática, a artéria gástrica esquerda e a artéria esplênica. 0 mesmo é descrito nos felinos domésticos (Ghoshal 1986). Diferentemente em suínos, a artéria celíaca apresenta como ramos terminais somente as artérias esplênica e hepática, sendo a artéria gástrica esquerda um ramo emitido pela artéria esplênica (Schwarze \& Schröder 1972, Ghoshal 1986).

A artéria mesentérica cranial foi o vaso de maior calibre em L. pardalis, surgindo caudalmente à artéria celíaca e seguindo em direção ao mesentério onde origina seus ramos. Quanto a sua origem, ventral a aorta e ao nível da segunda vértebra lombar, a mesma foi relatada nos animais domésticos, incluindo os gatos, bem como em mamíferos silvestres como o ouriço-cacheiro (Sphiggurus spp.) e o ratão-do-banhado (Myocastor coypus) (Ghoshal 1986, Machado \& Ziemman 2000, Machado et al. 2006).

Os ramos oriundos da artéria mesentérica cranial seguiram o padrão preconizado em carnívoros, diferenciando apenas na quantidade de artérias jejunais, que em L. pardalis resultaram em 12 unidades ao invés de 15 a 19 ramos (Ghoshal 1986).

A artéria adrenal, direita e esquerda, segundo Ghoshal (1986) podem ter origem na aorta abdominal ou na artéria renal, em nossos achados este vaso originou-se diretamen- te da aorta abdominal. Quanto às artérias renais, estas se dividem em ramos dorsais e ventrais antes de alcançar os rins, sendo duplas em $20 \%$ dos cães (principalmente a artéria renal esquerda) e em $10 \%$ nos gatos, antes da entrada na pelve (Nyland 2002), porém, esses achados não foram registrados em nosso estudo, destacando-se apenas o fato de que na fêmea de L. pardalis, a artéria renal esquerda tomou posição mais cranial em relação à direita, o que também difere do que é consagrado na literatura (Dyce 2010).

0 padrão de comportamento das artérias gonadais não diferiu do que está determinado nos carnívoros doméstico (Ghoshal 1986, Evans \& Lahunta 1994, König \& Leibich 2004, Dyce 2010).

A artéria mesentérica caudal da jaguatirica revelou conformidade semelhante ao descrito por Machado et al. (2008) e Schwarze \& Schröder (1972), os quais discorreram que nos carnívoros, a artéria mesentérica caudal é menor que a artéria mesentérica cranial, distribuindo-se fundamentalmente pelo cólon descendente e uma porção do reto, sendo o último ramo ímpar da aorta abdominal.

Como nos carnívoros em geral, a artéria mesentérica caudal de L. pardalis originou-se da face ventral da aorta abdominal (Ghoshal 1986, Evans \& Lahunta 1994, König \& Liebich 2004, Dyce 2010), entretanto estes autores nada abordam sobre a localização específica desta artéria em relação às outras, provenientes da aorta abdominal, enquanto, que na jaguatirica, foi constatada a sua formação ocorrendo após a artéria testicular direita, e imediatamente anterior a artéria circunflexa ilíaca profunda, percorrendo a aorta abdominal no sentido crânio-caudal.

Após sua origem, a artéria mesentérica caudal de $L$. pardalis deu início a uma bifurcação resultando na artéria cólica esquerda e artéria retal cranial, corroborando com resultados encontrados por Evans \& Christensen (1993) em estudos realizados em carnívoros.

$\mathrm{Na}$ jaguatirica, a artéria cólica esquerda apresentou calibre ligeiramente maior que a artéria retal, fato que, de acordo com Evans \& Lahunta (1994) não parece ser comum entre os carnívoros, uma vez que ambos os vasos apresentam calibre similar.

Verificou-se ainda, que na jaguatirica, a artéria cólica esquerda dirige-se cranialmente arqueando-se paralelamente ao cólon descendente, dividindo-se em 18 ramos. Estes, por sua vez, originam novos sub-ramos, que penetram na superfície do cólon descendente irrigando-o, corroborando com o abordado por König (2004), Frandson et al. (2005) e Dyce (2010) em carnívoros domésticos.

Posteriormente, a artéria cólica esquerda anastomosou-se com a artéria cólica média, um ramo da artéria mesentérica cranial, da mesma forma que o encontrado em estudos realizados por Carvalho et al. (1999) em cutia e por Machado et al. (2006) em ratão-do-banhado. Já a artéria retal cranial da jaguatirica percorre em direção caudal distribuindo oito ramos à porção final do cólon descendente e ao reto, até anastomosar- se com a artéria retal média, proveniente da artéria pudenda interna, do mesmo modo que ocorre em fetos de cães da raça Dogue Alemão (Campos et al. 2002) e em coelhos segundo Barone et al. (1996), que denominam esses ramos como artérias sigmóideas. 
Quanto à porção final da aorta abdominal, assim como nos felinos domésticos (Magno, et al. 2009), a jaguatirica revelou que tal vaso finaliza com a formação das artérias ilíacas (interna e externa) bem como a artéria sacral mediana.

\section{CONCLUSÃO}

A jaguatirica (Leopardus pardalis) possui vascularização arterial abdominal bastante semelhante ao descrito em felinos domésticos, com diferenças quanto ao número de artérias jejunais e origem das artérias renais.

Agradecimentos.- Agradecemos à Mina de Bauxita Paragominas-PA, pela doação dos animais ao Laboratório de Pesquisa Morfológica Animal (LaPMA) da Universidade Federal Rural da Amazônia (UFRA).

\section{REFERÊNCIAS}

Barone R. 1996. Anatomie Comparée des Mammifères domestiques Esplancnologie. Éditions Vigot, Paris, p.327-347.

Campos D.B., Silva F.O. \& Santos-Júnior M.J. 2002. Origens e ramificações das artérias mesentéricas cranial e caudal em caninos da raça dogue alemão. Hora Ciênc. 1(1):1-9.

Carvalho M.A.M., Miglino M.A., Dio L.J.A. \& Melo A.P.F. 1999. Artérias mesentéricas cranial e caudal em cutias (Dasyprocta aguti). J. Vet. Sci. 5(2): 17- 24.

Dyce K.M., Sack W.O. \& Wensing C.J.G. 2010. Tratado de Anatomia Veterinária. 4⿳a ed. Elsevier, Rio de Janeiro. 856p.

Emmons L.H. \& Feer F. 1990. Neotropical Rainforest Mammals: a field guid. University of Chicago Press, Chicago, p.151-152.

Evans H.E. \& Christensen G.C. 1993. Miller's Anatomy of the Dog. $3^{\text {rd }}$ ed. W.B. Saunders, Philadelphia. 1113p.

Evans H.E. \& Lahunta A. 1994. Abdome, pelve e membro pélvico, p.118119. In: Ibid. (Eds), Guia para a Dissecação do Cão. $3^{a}$ ed. Guanabara Koogan, Rio de Janeiro.

Frandson R.D., Wilke W.L. \& Fails A.D. 2005. Anatomia e Fisiologia dos Animais de Fazenda. $6^{\text {a }}$ ed. Guanabara Koogan, Rio de Janeiro. 454p.
Ghoshal N.G. 1986. Coração e artérias do carnívoro, p.1497-1550. In: Getty R. (Ed.), Sisson/Grossman's Anatomia dos Animais Domésticos. 5a ed. Guanabara Koogan, Rio de Janeiro.

International Committee on Veterinary Gross Anatomical Nomenclature 2012. Nomina Anatomica Veterinária. 5ae ed. Editorial Committee, Hannover. 160 p.

IUCN 2013. IUCN Red List of Threatened Species. Version 2013.2. <www. iucnredlist.org> Downloaded on March 20, 2014.

König H.E. \& Liebich H.G. 2011. In: Ibid. (Eds), Anatomia dos Animais Domésticos: texto e atlas colorido, órgãos e sistemas. $4^{\underline{a}}$ ed. Artmed, Porto Alegre. 768p.

Machado G.V. \& Ziemman F.P. 2000. Comportamento anatômico das artérias mesentéricas no ouriço-cacheiro (Sphiggurus spp. Cuvier, 1825). Anais Evento de Iniciação Científica da UFPR, Curitiba, PR, p.250. (Resumo)

Machado G.V., Gonçalves P.R., Parizzi A. \& Souza J.R. 2006. Padrão de divisão e distribuição das artérias mesentéricas no ratão-do-banhado (Myocastor coypus - Rodentia: Mammalia). Biotemas 19(1):59-63.

Machado M.R.F., Souza S.V. \& Oliveira T.C. 2008. Suprimento arterial dos intestinos do coelho da raça Nova Zelândia (Oryctolagus cuniculus). Biotemas 21(1):101-105.

Magno S.R., Pestana F.M. \& Hernandez J.M.F. 2009. Tronco celiaco-mesentérico em gato. Revta Port. Ciênc. Vet. 104:83-86.

Murray J.L. \& Gardner G.L. 1997. Leopardus pardalis. Mammalian Species 548:1-10.

Peduti-Neto J. \& Borelli V. 1970. Origem das artérias celíaca e mesentérica cranial, por tronco comum, em Felis catus domestica. Revta Fac. Med. Vet., São Paulo, 8:395-398.

Nyland T.G. \& Mattoon J.S. 2002. Small Animal Diagnostic Utrasound. $2^{\text {nd }}$ ed. W.B. Saunders Company, Philladelphia. 451p.

Oliveira T.G. \& Cassaro K. 1999. Guia de Identificação de Felinos Brasileiros. $2^{\underline{a}}$ ed. Sociedade de Zoológicos, São Paulo. 60p.

Schmidt D.P. \& Schoenau L.S.F. 2007. Origem das artérias celíaca e mesentérica cranial por tronco comum em cão. Ciência Rural 37(8):408411.

Schwarze E. \& Schröder L. 1972. Compendio de Anatomia Veterinária. Acribia, Zaragoza. 247p. 